\title{
Hydrophobic Shielding Drives Catalysis of Hydride Transfer in a Family of $\mathrm{F}_{420} \mathrm{H}_{2}$-Dependent Enzymes
}

\author{
A. Elaaf Mohamed, ${ }^{a}$ Karmen Condic-Jurkic, ${ }^{a}$ F. Hafna Ahmed, ${ }^{a}$ Peng Yuan, ${ }^{a}$ Megan L. O’Mara, ${ }^{\text {a }}$ Colin J. Jackson*a and Michelle \\ L. Coote*a,b
}

aResearch School of Chemistry, Australian National University, Canberra, ACT 2601, Australia

${ }^{\mathrm{b}} \mathrm{ARC}$ Centre of Excellence for Electromaterials Science

KEYWORDS: Pretomanid, Ddn, Deazaflavin-dependent nitroreductase, F420, F420H2, hydrophobic shielding

\begin{abstract}
A family of flavin/deazaflavin-dependent oxidoreductases (FDORs) from mycobacteria has been recently characterized and found to play a variety of catalytic roles, including the activation of prodrugs such as the candidate anti-tuberculosis drug, pretomanid (PA-824). However, our understanding of the catalytic mechanism used by these enzymes is relatively limited. To address this, in the present work we have used a combination of quantum mechanical and molecular dynamics calculations to study catalytic mechanism of the activation of pretomanid by the deazaflavin-dependent nitroreductase (Ddn) from Mycobacterium tuberculosis. The preferred pathway involves an initial hydride transfer step from the deprotonated cofactor (i.e. $\left.\mathrm{F}_{420} \mathrm{H}^{-}\right)$, with subsequent protonation, before undergoing a series of spontaneous intramolecular reactions to form the final reactive nitrogen species. The most likely proton source is a hydroxonium ion within the solvent accessible active site. Intriguingly, catalysis of the ratedetermining hydride transfer step is aided by three tyrosine residues that form a hydrophobic barrier around the active site that, upon reaction, is then disrupted to allow increased water accessibility to facilitate the subsequent proton transfer step. The catalytic mechanism we propose is consistent with previous experimental observations of the Ddn enzyme, and will inform the design of improved pro-drugs in the future.
\end{abstract}

\section{INTRODUCTION}

Tuberculosis (TB) is the leading bacterial cause of death, with over 1.5 million deaths due to TB in 2014 alone. ${ }^{1}$ TB is caused by the highly infectious air-borne bacterium Mycobacterium tuberculosis, which is able to evade the host immune system and persist in a dormant, or latent, state in "tubercles" in the lungs for many years. ${ }^{2}$ A third of the world's population is thought to carry dormant $M$. tuberculosis, which can cause active infection when individuals become immunocompromised, for instance due to HIV infection or diabetes. ${ }^{1,3,4}$ The ability of $M$. tuberculosis to persist in a latent state, during which it is highly resistant to antibiotics, has contributed to the emergence of multi-, total- and extensively drug-resistant strains that cannot be eliminated by currently available drugs. ${ }^{5}$

After several decades without the introduction of novel treatments for TB, bicyclic-nitroimidazole pro-drugs were found to have anti-mycobacterial activity in $2000 .^{6}$ Three of these compounds are currently either in late-stage clinical trials, or are approved for use against multi-drug resistant infection, namely pretomanid (previously PA-824, structure shown in Figure 1), delamanid (previously TBA-354) and OCP-67683. ${ }^{7,8}$ The mechanism of action of these drugs is not fully understood, although it is known that they are reductively activated in $M$. tuberculosis. The main hypothesis is that reductive activation leads to release of reactive nitrogen species that contributes to bacterial cell death. ${ }^{9,10}$ In addition, the des-nitro products can inhibit mycolic acid synthesis, which is required to generate the robust mycobacterial cell wall, ${ }^{10}$ making them ideal for use in combination drug therapy to increase mycobacterial susceptibility to other drugs.
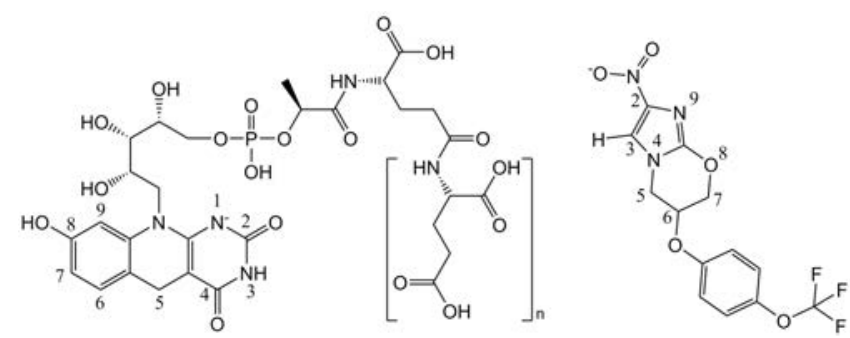

Figure 1. Structure of the cofactor $\mathrm{F}_{420} \mathrm{H}^{-}$and pretomanid. The cofactor $\mathrm{F}_{420} \mathrm{H}^{-}$acts as a reducing agent for pretomanid in the reaction catalysed by Ddn.

The reduction of pretomanid in M. tuberculosis is carried out by an enzyme belonging to the flavin/deazaflavin dependent oxidoreductase (FDOR) superfamily, known as the deazaflavin-dependent nitroreductase (Ddn). ${ }^{9,11}$ Ddn utilizes the deazaflavin cofactor $\mathrm{F}_{420} \mathrm{H}_{2}$ as an electron source, although the deprotonated species (i.e., $\mathrm{F}_{420} \mathrm{H}^{-}$) is thought to be the active species (Figure 1). ${ }^{12}$ The reduced $\mathrm{F}_{420}$ species undergoes obligate 2-electron transfer with a measured redox potential of $340 \mathrm{mV} .{ }^{13} \mathrm{~F}_{420} \mathrm{H}_{2}$ is generated from $\mathrm{F}_{420}$ in mycobacteria by the enzyme $\mathrm{F}_{420}$-dependent glucose-6-phosphate dehydrogenase (FGD). ${ }^{14}$ Thus FGD serves to regenerate $\mathrm{F}_{420} \mathrm{H}_{2}$ for each catalytic cycle following formation of $\mathrm{F}_{420}$ during pretomanid activation. The final products resulting from reduction of pretomanid by Ddn were characterized experimentally, ${ }^{9}$ identi- 
fying the major products as des-nitroimidazole and nitrous acid, which can readily decompose and lead to nitric oxide formation. ${ }^{9}$

Chemical reduction of pretomanid using sodium borohydride as an analogous hydride donor to $\mathrm{F}_{420} \mathrm{H}_{2}$ showed an initial hydride transfer to $\mathrm{C} 3$ of pretomanid followed by protonation of the intermediate $\mathrm{C} 2$ by the solvent. ${ }^{9}$ However, the full mechanism of pretomanid reduction by $\mathrm{F}_{420} \mathrm{H}_{2}$ and Ddn has not been characterized in detail and the role of the enzyme is poorly understood.

The crystal structure of Ddn in complex with the oxidised $F_{420}$ cofactor was solved in 2012, providing important insights into binding mode of the cofactor. ${ }^{15}$ The structure showed that the cofactor is bound with its $R e$-face oriented towards the substrate binding site. ${ }^{15}$ Several residues surrounding the cofactor have been identified as being catalytically relevant through mutagenesis studies and substrate docking analysis. ${ }^{15}$ However, a crystal structure of the ternary complex with both cofactor and substrate is not available. The residues suggested to participate in binding of pretomanid and $\mathrm{F}_{420}$ in the active site include four tyrosines (Tyr65, Tyr130, Tyr133 and Tyr136) and a serine (Ser78). Their potential roles in the reaction mechanism are currently unknown, but mutations of these residues resulted in the loss of pro-drug reduction activity.

The aim of our study was to elucidate the catalytic mechanism for the activation of pretomanid by Ddn and $\mathrm{F}_{420} \mathrm{H}_{2}$ using computational modelling. As noted above, our previous study has established that the reduced cofactor bound to Ddn is likely to be in its deprotonated form $\mathrm{F}_{420} \mathrm{H}^{-}$(Figure 1). ${ }^{12}$ Hence, this protonation state was used in our mechanistic study. We used docking to predict the possible binding modes of pretomanid and molecular dynamics (MD) simulations to validate the stability of the selected docked structure. MD simulations were also used to gain insights in the molecular interactions between the cofactor and substrate, and the surrounding protein residues. Finally, we used density functional theory (DFT) calculations, benchmarked against high-level $a b$ initio methods, to characterize the reaction profile for redox activation of pretomanid by $\mathrm{F}_{420} \mathrm{H}^{-}$using a small model system of the active site. This study is the first computational investigation of the proposed Ddn mechanism. Here we implemented a stepwise approach to investigate different mechanistic possibilities while keeping the computational costs tractable. This study provides useful insights into the catalytic mechanism employed by Ddn and creates a firm foundation for future mechanistic studies of Ddn and other $\mathrm{F}_{420}$-dependent FDOR superfamily members.

\section{METHODS}

\subsection{Reconstruction of the N-terminus and Docking}

Although the N-terminus of Ddn is required for enzyme activity, its presence promotes protein aggregation which prevented structural characterization of the full-length protein. ${ }^{15}$ Hence, the available crystal structures of Ddn represent an inactive, $\mathrm{N}$-terminally truncated form of the protein. ${ }^{15}$ Here we used the crystal structure of the Ddn: $\mathrm{F}_{420}$ complex (PDB ID: 3R5R) solved at $1.80 \AA$ resolution, in which the N-terminal 40 residues of the protein are missing. ${ }^{15} \mathrm{~N}$-terminus is expected to participate in substrate binding, hence, a homology model of the N-terminus was developed using SWISS-MODEL to enable a meaningful modelling of the ternary enzyme complex formed during the reaction ${ }^{16-19}$. The homology model of the complete Ddn sequence was developed based on the fulllength structure of a closely related homolog from Nocardia farcinica (PDB ID: 3R5Y) ${ }^{15}$ containing a helical N-terminal domain. The $\mathrm{C} \alpha$ root mean square deviation (RMSD) between the conserved regions of the Nocardia farcinica enzyme structure and that of the Mycobacterium tuberculosis Ddn structure is $0.95 \AA$. The resulting homology model of the helical Nterminal domain was then incorporated into the structure of the truncated-Ddn: $\mathrm{F}_{420}$ complex, maintaining the same orientation as observed in the Nocardia farcinica template structure. This chimeric structure was then subjected to a steepest descent energy minimization using GROMACS 4.6.5 in conjunction with GROMOS 54A7 force field. ${ }^{20}$ The energy minimized structure was used to dock pretomanid into the substrate binding site using Autodock Vina, where both substrate and protein were prepared using default settings on AutodockTools. $^{21,22}$

\subsection{Molecular Dynamics Simulations}

In our previous work, we used a combination of experimental and computational methods to determine that the cofactor $\mathrm{F}_{420} \mathrm{H}_{2}$ is bound to Ddn in its deprotonated form with N1 losing its hydrogen. ${ }^{12}$ Parameters for deprotonated $\mathrm{F}_{420} \mathrm{H}^{-}$and for pretomanid compatible with the GROMOS 54A7 force field were obtained through the Automated Topology Builder (ATB entries: 28649 and 28646 respectively). ${ }^{23,24}$ Protonation states of titratable residues were estimated with $\mathrm{H}++$ server, ${ }^{25}$ resulting with the standard protonation states for titratable residues at pH 7 (no histidines are present in Ddn). Molecular dynamics (MD) simulations were performed under periodic boundary conditions with the ternary complex (Ddn-pretomanid- $\mathrm{F}_{420} \mathrm{H}^{-}$) placed in a cubic box with at least $1.6 \mathrm{~nm}$ distances between the protein and the box walls in all directions. Van der Waal's forces and Coulombic interactions were evaluated using a 1.4 $\mathrm{nm}$ cut-off scheme for both with a corrective reaction-field applied using a dielectric constant of $\varepsilon_{\mathrm{r}}=78.5$ for long-range electrostatic interactions beyond the cut-off distance. A time step of $2 \mathrm{fs}$ was used and the LINCS algorithm was applied to constrain all the bond lengths. ${ }^{26}$ The simulations were carried out at temperature of $300 \mathrm{~K}$ and pressure of 1 bar using Berendsen weak-coupling method with time constants of 0.1 and 0.4 ps, respectively. ${ }^{27}$

The simulation box was solvated along with the ternary complex using SPC water and $3 \mathrm{Cl}^{-}$counter ions were added to make the system neutral overall. ${ }^{28}$ The energy of the system was then minimized via steepest descent to relax the solvent while the ternary complex was restrained with force constants of $1000 \mathrm{~kJ} \mathrm{~mol}^{-1} \mathrm{~nm}^{-1}$. These restraints were then gradually lowered by performing a series of four consecutive simulations each lasting $2 \mathrm{~ns}$ with force constants of $1000 \mathrm{~kJ} \mathrm{~mol}^{-1}$ $\mathrm{nm}^{-1}, 500 \mathrm{~kJ} \mathrm{~mol}^{-1} \mathrm{~nm}^{-1}, 200 \mathrm{~kJ} \mathrm{~mol}^{-1} \mathrm{~nm}^{-1}$, and $50 \mathrm{~kJ} \mathrm{~mol}^{-1}$

$\mathrm{nm}^{-1}$, respectively. Finally, two independent $50 \mathrm{~ns}$ production runs with no restraints were carried out. An additional $50 \mathrm{~ns}$ simulation containing $\mathrm{F}_{420}$ and the negatively charged pretomanid intermediate (ATB entries: 29710 and 29334 respectively) formed following hydride transfer was also performed to investigate changes in solvent accessibility.

The ternary state trajectories were concatenated prior to analysis. The protein conformations were aligned using the leastsquare fitting of the backbone atoms to the reference (initial) 
structure. Cluster analysis was performed using the method by Daura et $\mathrm{al}^{29}$ to identify the predominant protein conformations during the simulations. In this method, the protein conformations were clustered based on the pairwise RMSD. Two conformations were considered to belong to the same cluster if their RMSD was lower than the specified cut-off value of $2.0 \AA$. The simulation results were visualized and images produced using VMD. ${ }^{30}$

\subsection{Definition of the Quantum Mechanical Model Systems}

It has been postulated that the major role of the enzyme is to align the cofactor $\mathrm{F}_{420} \mathrm{H}^{-}$and pretomanid in the specific configuration to allow the reaction to proceed. ${ }^{15}$ According to the proposed mechanism, surrounding residues in the active site are not involved directly in catalysis, but providing a favourable environment. Currently, it is not yet possible to model the entire protein with QM methods, imposing a limit to the system size subjected to QM calculations. Instead, chemical reactions are usually modelled by employing only reactants and the environment is accounted for implicitly through continuum models. The similar strategy is often used to model enzyme reactions by building minimalistic model systems that include only the chemically active groups to capture relevant reaction steps, while excluding the environment. This allows a crude characterization of stationary points on the potential energy surface, often revealing the crucial steps in the mechanism. Additionally, the usage of small model systems allows benchmarking against more accurate high-level quantum mechanical (QM) methods before engaging in the more elaborate methods that are able to include the environment effects to an extent, such as a cluster approach or QM/MM. ${ }^{31}$ We used the small models to validate the performance of several different DFT functionals for this reaction against the high-level G3(MP2)-CC method (see Section 2.4).

Another commonly used approach is so-called cluster approach, which relies on larger models that include all the active site residues and sometimes more. Although this approach has been successfully applied in a number of enzyme studies, it relies heavily on the representation of the active site present in the crystal structure. Namely, to allow calculation with such large models, the coordinates of $\mathrm{C}$ atoms are usually kept frozen. In addition, the system size increases the available conformational space, hence, increasing complexity of the potential energy surface.

In the present work, the use of quantum cluster models allowed us to evaluate several different pathways for Ddn catalytic processes with accurate calculations but at a relatively low computational cost, so as to provide a basis for the design of future QM/MM studies. The reaction can be broken down in three steps (Scheme 1) - hydride (Step 1) and proton transfer (Step 2), followed by the subsequent decomposition of the reduced pretomanid intermediate (Step 3). Each step was studied separately and two alternate small model systems were employed in these calculations. The models were designed to include all the reactive species (cofactor, pretomanid, proton source) relevant for the specific step. The variations of each basic model were introduced to investigate the effect of the proton donor in Step 2 on the reaction profile, as well as the role of Ser78 in Step 3.

Model 1. This model is used to study first two steps of the mechanism comprising the hydride transfer (Step 1) and subsequent proton transfer (Step 2). It consists of $\mathrm{F}_{420} \mathrm{H}^{-}$, preto- manid and a proton donor. The representation of the cofactor and substrate was simplified by truncating distal regions of the molecules important for binding, but not contributing directly to chemical reaction. Hence, the ribityl-lactyl-polyglutamate tail in $\mathrm{F}_{420} \mathrm{H}^{-}$and trifluoromethoxy-benzyl tail moiety in pretomanid was replaced with a methyl group and a hydrogen atom to further reduce the system size. To study the effect of the proton donor on the energy barrier describing the proton transfer in the second step of the mechanism, the model described above was modified accordingly. We used four different proton donors - hydroxonium ion $(\mathbf{A})$; neutral water molecule $(\mathbf{B})$; water molecule partially protonated by phenol $(\mathbf{C})$; and phenol alone (D). The phenol molecule was a substitute for tyrosine Tyr130, which has been previously assumed to assist in proton transfer.

Model 2. This model removed the oxidized $F_{420}$ cofactor formed following hydride transfer and was used to evaluate different proton donors for the proton transfer step (Step 2).

Model 3. This model was used to study intramolecular decomposition producing the final products des-nitro metabolite and nitrous acid (Step 3). It is assumed that once the hydride and proton are transferred successfully to form the reduced pretomanid intermediate, $\mathrm{F}_{420}$ and the proton donor are free to regenerate for the next catalytic cycle. Hence, Model 3 includes only pretomanid, while Model 3A contains an additional ethanol molecule as a serine analogue. It was found in MD simulations that Ser78 forms a hydrogen bond with the leaving $\mathrm{NO}_{2}$ group, which might facilitate this reaction step.

\subsection{Computational Details}

A benchmarking assessment was initially performed on a truncated system that featured similar hydride and proton transfer reactions with the important functional groups kept intact (SI Figure S1.1, SI Table S1). This study used the high-level composite $a b$ initio molecular orbital theory method G3(MP2)-CC as a benchmark against which other functionals were then compared. ${ }^{32,33}$ The functionals tested were B3LYP, M06-2X, M06-HF, M06-L and M11 in conjunction with the $6-31+\mathrm{G}(\mathrm{d}, \mathrm{p})$ basis set. On average, the relative energies computed with the M06-2X functional were in the best agreement with G3(MP2)-CC results. Thus, for this study, all QM calculations were performed at M06-2X/6-31+G(d,p) level of theory using the GAUSSIAN09 suite of programs. ${ }^{34}$ Since the active site of the enzyme is a solvent accessible cleft in the protein, the calculations were performed in the aqueous solvent phase using the SMD solvent model including for the geometry optimizations. ${ }^{35}$ The calculations for the hydride transfer step were repeated in a low polarity environment to simulate the effect of hydrophobic shielding. This was achieved by using toluene as a solvent in the SMD model. The calculated transition states were confirmed with IRC (Intrinsic Reaction Coordinate) calculations using the HPC method. ${ }^{36,37}$

\section{RESULTS}

\subsection{The Binding Mode and Enzyme-Substrate Complex}

As it is essential for activity, the N-terminus of Ddn was reconstructed based on the closely related $(0.95 \AA \mathrm{C} \alpha \mathrm{RMSD}$ for the enzyme) homolog from $N$. farcinica (PDB ID: 3R5Y). ${ }^{15}$ Using Autodock Vina, ${ }^{21}$ the lowest free energy binding mode of pretomanid to this full-length Ddn structural model $(-8.2$ kcal. $\mathrm{mol}^{-1}$ ) is similar to the mode previously predicted for the 
truncated protein complex. ${ }^{15}$ The $\mathrm{F}_{420}$ cofactor was bound with the $R e$ face towards the substrate in the crystal structure and held in place by hydrogen bonding between the $\mathrm{C} 4$ carbonyl oxygen of the isoalloxazine chromophore and the hydroxyl group of Tyr133. Hydrogen bonding between the phospholactate and polyglutamate tail moieties and the enzyme further contributed to the cofactor positioning. The nitroimidazole group of the docked pretomanid formed a hydrogen bond between the $\mathrm{NO}_{2}$ group and Ser78, while the trifluoromethoxybenzyl tail was largely stabilized by $\pi-\pi$ interactions with Trp20 and Phe41, as shown in Figure 2. The involvement of Trp20 is consistent with results of previous site-directed mutagenesis studies, which showed that mutants lacking Trp20 exhibit decreased affinity towards pretomanid. ${ }^{15}$ The phenylalanine residues Phe16 and Phe17 were not directly involved in this binding mode, despite showing limited contribution towards substrate affinity. ${ }^{15}$

MD simulations were carried out to investigate protein dynamics and interactions in the active site using the structure with docked pretomanid as the starting point. The simulations were performed for a total of $100 \mathrm{~ns}$ ( 2 x $50 \mathrm{~ns}$ trajectories $)$, during which time the movement of $\mathrm{F}_{420} \mathrm{H}^{-}$, pretomanid and the catalytically important residues - Tyr65, Ser78, Tyr130, Tyr133 and Tyr136, were studied along with the interactions between these groups. The average RMSD for the entire protein backbone was $1.6 \pm 0.3 \AA$ with respect to the initial structure, indicating a high degree of conformational stability over the course of simulations, including the reconstructed N-terminus region. The average backbone RMSD of the reconstructed first 40 residues only amounted to $3.3 \pm 0.9 \AA$, which reflects high- er local fluctuations characteristic for termini and coil regions. As demonstrated in Figure 2 (top), the reconstructed Nterminus is fairly stable, with some minor changes in the helix orientation and relaxation to coil on both ends of the helix. This does not represent a significant structural change, and suggests that the homology model was a reasonable approximation of the missing part of the structure. The cofactor and docked substrate remained stably bound in the active site throughout the simulations with the average RMSDs of $2.6 \pm$ $0.8 \AA$ and $1.4 \pm 0.4 \AA$, respectively.

The oxidized form of the $\mathrm{F}_{420}$ cofactor present in the crystal structure was replaced by the deprotonated form $\mathrm{F}_{420} \mathrm{H}^{-}$in the MD simulations, the latter being the form most likely present in catalysis. ${ }^{12}$ The hydrogen bonding between the cofactor and Tyr133 was observed to be stable throughout the duration of the simulation. In contrast, Tyr65, which was also proposed to be involved in cofactor binding, ${ }^{15}$ did not exhibit any direct binding interactions with $\mathrm{F}_{420} \mathrm{H}^{-}$. The $\mathrm{NO}_{2}$ group of pretomanid and the hydroxyl of Ser78 formed a strong hydrogen bond as predicted by the in silico docking, which was maintained over the course of the simulation. The second anchor point of pretomanid, the $\pi-\pi$ interactions between its trifluoromethoxybenzyl tail and the N-terminus residues Trp20 and Phe41 was more flexible with the tail showing occasional movement that extended beyond the two residues with intermittent interactions with Phe16 and Phe17 in agreement with the mutagenesis results. ${ }^{15}$ 


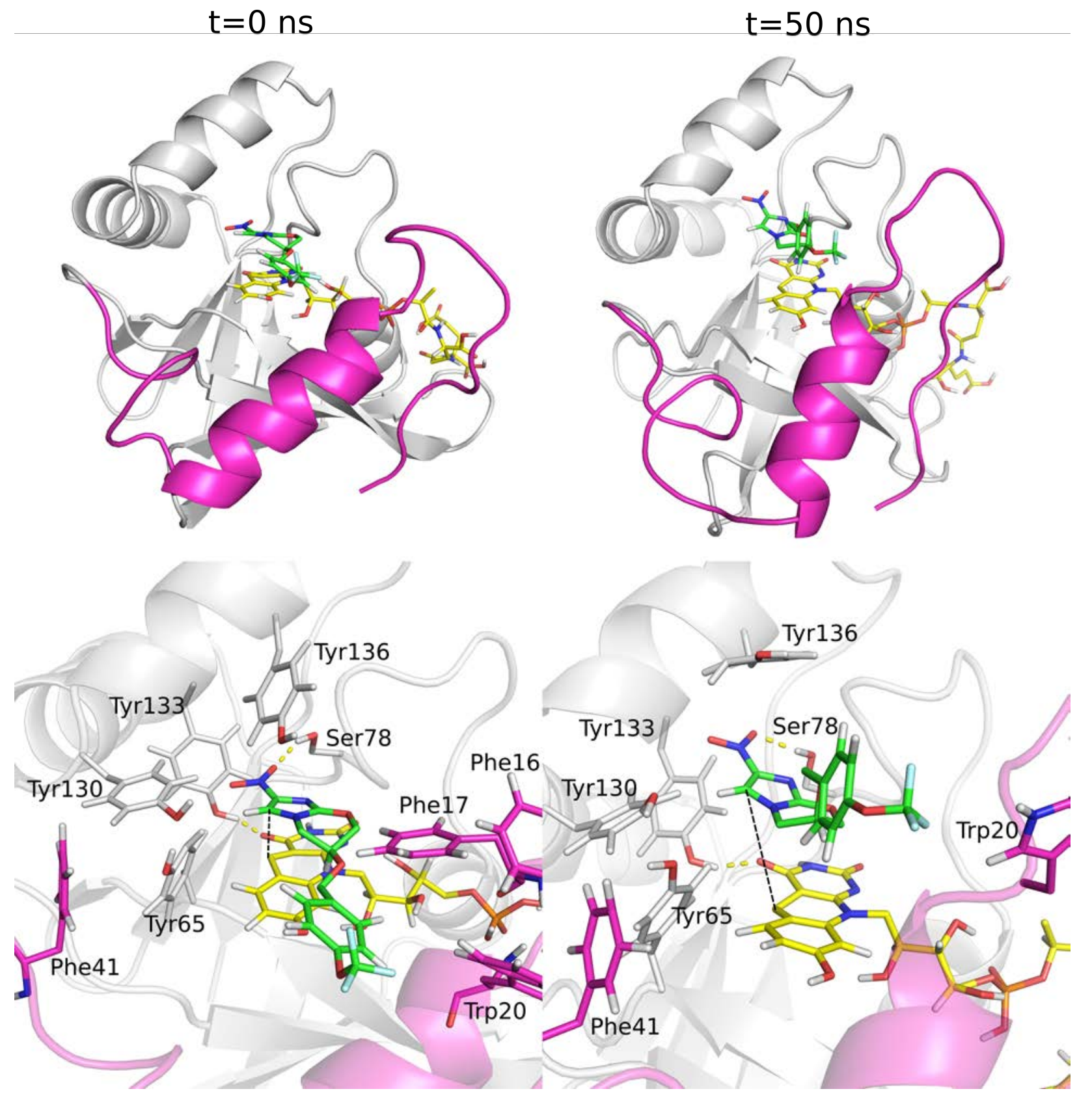

Figure 2. Top. The overall holoenzyme structure at the start and end of the MD simulations from left to right. The residues and backbone in magenta are from the reconstructed N-terminus while white represents the published crystal structure. ${ }^{15}$ Pretomanid is shown in green while $\mathrm{F}_{420}$ is shown in yellow. Bottom. A closer look at the Ddn active site and the interactions of $\mathrm{F}_{420} \mathrm{H}^{-}$and pretomanid with the surrounding active site residues at the in the starting and final MD coordinates from left to right. The average $\mathrm{C} 5$-C3 distance, marked with dashed line, as measured in MD simulations was $4.5 \pm 0.5 \AA$.

The relative position of the cofactor and pretomanid during the simulations is of significance from a mechanistic perspective. Namely, the first step of the reaction involves the transfer of the hydride from the $\mathrm{C} 5$ atom of $\mathrm{F}_{420} \mathrm{H}^{-}$to the $\mathrm{C} 3$ of the pretomanid. For this transfer to occur, $\mathrm{F}_{420} \mathrm{H}^{-}$and pretomanid need to be favourably oriented and the relevant carbon atoms in close proximity. The initial configuration of the cofactor and the docked pretomanid in the active site resulted in a structure in which the aromatic rings of the reactants are stacked, with the pretomanid $\mathrm{C} 3$ atom positioned directly above the $\mathrm{F}_{420} \mathrm{H}^{-}$ $\mathrm{C} 5$ atom. This binding mode was maintained throughout the simulations and the average $\mathrm{C} 5-\mathrm{C} 3$ distance measured in the MD simulations was $4.5 \pm 0.5 \AA$. This distance is likely to correspond to the formation of the early-stage Michaelis com- plex, which can then lead to the successful hydride transfer. A distance of $3.3 \AA$ was calculated in the reaction complex (RC) prior to the hydride transfer step using DFT calculations on the small model system. The $\mathrm{C} 5-\mathrm{C} 3$ distance characterizing the reaction complex will vary depending how close this structure is to the transition state $(2.7-3.1 \AA),{ }^{38-40}$ which is not possible to observe in the MD simulations due to classical treatment of the interactions. However, the stability of the complex during the simulation and a reasonable distance between the reactants provides sufficient support for feasibility of the suggested mechanism.

In addition to helping ascertain the reaction complex of pretomanid: $\mathrm{F}_{420} \mathrm{H}^{-}$, the in silico docking and $\mathrm{MD}$ simulations also presented viable alternative binding modes for preto- 
manid within the holoenzyme active site that were not conducive to the presented mechanism. One mode in particular, with pretomanid flipped $180^{\circ}$ along its axis, similar to the $R$ enantiomer of pretomanid, ${ }^{41}$ exhibited improved hydrogen bonding to Tyr130. Although this conformation was not beneficial to the mechanism in this study, due to misalignment of the C5-C3 hydride transfer path, it might provide additional insight into future drug development and a brief analysis is included in the supporting information (SI S2).

\subsection{Roles of the Active Site Residues}

The roles of the active site residues - Tyr65, Ser78, Tyr130, Tyr133 and Tyr136 - were further examined using MD simulations. The hydroxyl group of Ser78 formed a strong hydrogen bond to the $\mathrm{NO}_{2}$ head group of pretomanid, which was maintained throughout the simulations. Similarly, a hydrogen bond between Tyr133 and the $\mathrm{C} 4$ carbonyl group of $\mathrm{F}_{420} \mathrm{H}^{-}$ was observed during the simulations. However, two tyrosine residues, previously suggested to have a role in stabilizing the $\mathrm{F}_{420}$ complex, Tyr65 and Tyr130, ${ }^{15}$ did not form any direct interactions with pretomanid or $\mathrm{F}_{420} \mathrm{H}^{-}$in either the docking result or in the subsequent MD simulations. Instead, they were only observed to interact occasionally with other nearby residues, including each other. Moreover, Tyr136 was previously predicted to interact with pretomanid or $\mathrm{F}_{420} \mathrm{H}^{-}$via its hydroxyl group. ${ }^{15}$ However, the MD simulations showed that the residue is actually aligned with pretomanid in an orientation that most likely facilitates $\pi-\pi$ interactions between the aromatic phenol ring of Tyr136 and the nitroimidazole group of pretomanid.

The MD simulations suggest that these three tyrosine residues play a different role than that predicted by the previous structural study. ${ }^{15}$ Rather than form hydrogen bonds with pretomanid or $\mathrm{F}_{420} \mathrm{H}^{-}$, Tyr65, Tyr130 and Tyr136 were observed to form a hydrophobic barrier around the site of hydride transfer and the $\mathrm{NO}_{2}$ head group of pretomanid in the Michaelis complex. This barrier was maintained through intermittent hydrogen bonding interactions between the hydroxyl groups of the tyrosine residues. Previously, mutagenesis of these residues showed that loss of the aromatic ring in any of them resulted in complete loss of activity whereas the mutation to phenylalanine, with the aromatic ring left intact, only resulted in partial loss in activity. ${ }^{15}$ Our simulations suggest that this partial loss of activity can be explained by the inability of the mutants to form hydrogen-bonding interactions important for formation and stabilization of the hydrophobic barrier.

\subsection{The Reaction Mechanism}

The overall activation reaction can be broken down into three discrete steps. These steps include the hydride transfer from $\mathrm{F}_{420} \mathrm{H}^{-}$to pretomanid (Step 1), followed by the proton transfer to the reduced pretomanid (Step 2), and finally, the spontaneous intra-molecular decomposition reaction to form desnitroimidazole and release reactive nitrous acid species (Step 3 ), as shown in Scheme 1. We used a small model approach to characterize potential surface for each step in a modular fashion, which has enabled us to test four different potential proton donors in the proton transfer (Step 2). In addition, we examined the effect of hydrogen bonding between Ser78 and nitro group of pretomanid, observed in MD simulations, on the barrier for the intra-molecular decomposition (Step 3). Ser78 was mimicked with an ethanol molecule.
Scheme 1. The overall activation mechanism.<smiles>O=[N+]([O-])c1nc2n(c1[O-])CCCO2</smiles>

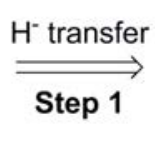

Step 3<smiles>O=NO</smiles>

decomposition

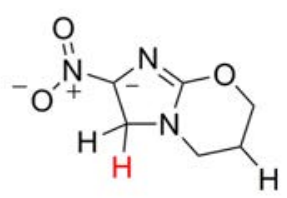

Step $2 \Downarrow \mathrm{H}^{+}$transfer

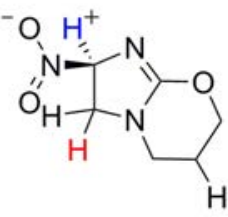

Benchmarking of the functionals (Methods 2.4) showed an average error of $36.3 \mathrm{~kJ} \mathrm{~mol}^{-1}$ in B3LYP, which is commonly used in QM/MM calculations as the QM level of theory. Comparatively, M06-2X yielded an average error of $11.9 \mathrm{~kJ}$ $\mathrm{mol}^{-1}$, which is a marked improvement over B3LYP. A quantum cluster approach using M06-2X/6-31+G(d,p) was therefore thought to provide better overall accuracy, and was used to characterize the stationary points on the potential energy surface for the mechanism suggested in Scheme 1. Benchmarking is an important component of computational studies, but it is often neglected in the studies of biological systems due to computational limitations because of large system sizes. The implementation of small model systems allowed us to select appropriate functional for future studies, while obtaining valuable information about mechanistic steps of Ddn reaction. Calculations for Step 1 were performed using Model 1, which includes the reaction complex (RC) of deprotonated $\mathrm{F}_{420} \mathrm{H}^{-}$ and pretomanid in their truncated form, and a proton donor required to complete the reduction (see Methods 2.3). The resulting optimized $\mathbf{R C}$ structure and the subsequent intermediates and transition states for the hydride/proton transfer step are presented in Figure 3, where the proton donor is represented by a hydroxonium ion.

\subsection{Step 1: Hydride Transfer}

The first step in the activation mechanism is the hydride transfer step. Hydride transfer takes place from the $\mathrm{C} 5$ atom of the deprotonated $\mathrm{F}_{420} \mathrm{H}^{-}$to the $\mathrm{C} 3$ atom of pretomanid. Here the relative energies are calculated with respect to the RC complex, which is set to zero (Figure 3). The relative energy of transition state (TS1) for this hydride transfer is $95.8 \mathrm{~kJ} \mathrm{~mol}^{-1}$ higher than that of RC. The C5-C3 distance of $3.3 \AA$ in the RC was decreased to $2.7 \AA$ in TS1. The intermediate complex (IC1) formed as the product of the hydride transfer step is -9.2 $\mathrm{kJ} \mathrm{mol}^{-1}$ lower than RC. IC1 consists of a negatively charged intermediate species of pretomanid and the oxidized cofactor $\mathrm{F}_{420}$, which is then available to be reduced for the next catalytic cycle. In this part of catalytic cycle, the proton donor (pictured as a hydroxonium ion in Figure 4) acts as a spectator. Proton transfer takes place upon formation of IC1.

Considering that the hydrophobic shielding by the tyrosine residues are in effect during the hydride transfer step, it is also imperative to compare the reaction barrier at a lower dielectric constant with the system in aqueous. Therefore, the SMD solvent model was used with toluene as a solvent to emulate hy- 
drophobic shielding effect, and the relative energy of the transition state (TS1) was calculated to be $117.3 \mathrm{~kJ} \mathrm{~mol}^{-1}$ higher in energy than RC. Meanwhile, the resulting intermediate complex (IC1) was determined to be $68.4 \mathrm{~kJ} \mathrm{~mol}^{-1}$ higher than the starting $\mathbf{R C}$ complex.

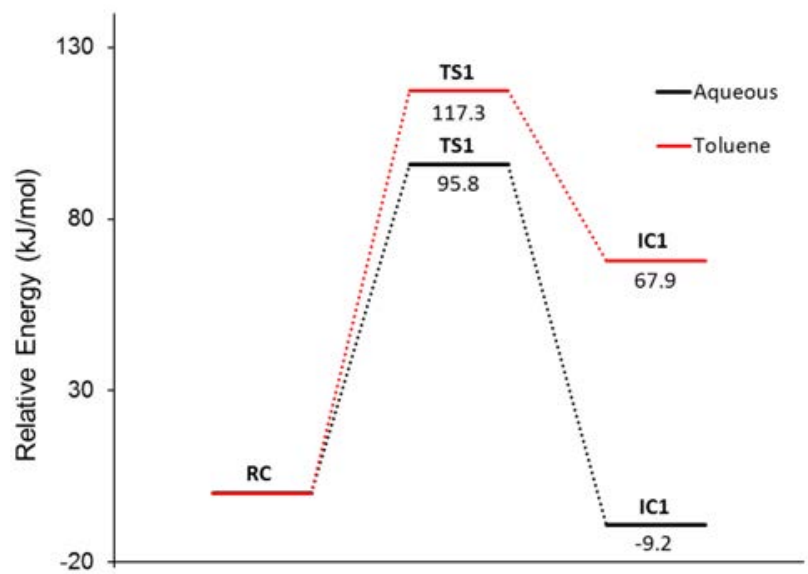

Figure 3. Comparison of the reaction profiles for hydride transfer step in water and toluene. All values are given in $\mathrm{kJ}$ $\mathrm{mol}^{-1}$.

Both water and toluene represent two extremes in the representation of the active site environment, as it is neither purely hydrophobic nor entirely aqueous. Interestingly, the change of the solvent had a stronger impact on the relative energies of the intermediate IC1 than on the reaction barrier, while the number of charged species did not change. As discussed later, the results suggest that the hydrophobic shield does not necessarily not have a major impact on the barrier height per se but rather acts to protect the species from solvent. Moreover, upon its disruption the aqueous environment significantly stabilizes the product preventing the reverse reaction from occurring and driving the process forward.

\subsection{Step 2: Proton Transfer}

Since $\mathrm{F}_{420} \mathrm{H}^{-}$is present in its deprotonated state, ${ }^{12}$ it cannot act as a proton donor in the second step of the reaction. Its role in the reaction is complete with the formation of the oxidized $\mathrm{F}_{420}$ species, following the first hydride transfer step. Therefore, another proton donor is required for successful completion of the reduction reaction. Four reaction mechanisms were tested, involving three different species as proton donors that are shown in Scheme 2. The four reaction mechanisms are: (A) direct proton transfer from a hydroxonium ion; (B) direct proton transfer from a neutral water molecule; (C) direct proton transfer from the hydroxyl group of a tyrosine; (D) proton transfer from a neutral water molecule assisted by a tyrosine. Figure 4 compares the energy profiles of these four mechanisms, along with the optimized geometries of their respective transition states. The starting intermediate complex in this comparison, IC1, is considered equivalent in all four mechanisms as differences in proton donors do not influence the preceding hydride transfer step. The relative energies were calculated with respect to IC1 using Model 2.

Scheme 2. The four proton transfer reaction mechanisms.

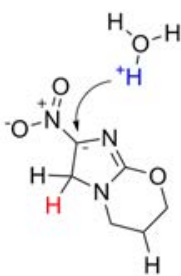

(A)

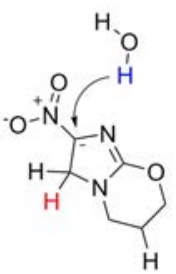

(B)

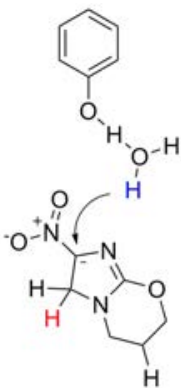

(C)

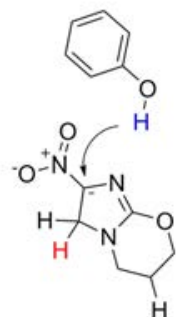

(D)
Mechanism (A). The first mechanism uses a hydroxonium ion as a proton donor. The transition state TS1 is close to barrierless with a barrier of just $13.8 \mathrm{~kJ} \mathrm{~mol}^{-1}$ above IC1. The reaction is also highly exothermic $\left(-99.1 \mathrm{~kJ} \mathrm{~mol}^{-1}\right)$. It is worth noting that the energy cost of forming the hydroxonium ion was not taken into account when comparing to the alternate proton transfer reactions. However, the extra proton could readily propagate from the bulk solvent where protons would be in excess. Furthermore, deprotonation of the initial $\mathrm{F}_{420} \mathrm{H}_{2}$ molecule could serve as a potential source of protons excess in the vicinity of the active site due to its pKa of 6.9 and its preferred binding protonation state. ${ }^{12}$ Similar reaction mechanisms have been characterized in literature utilizing hydroxonium ions as proton donors generated from the surrounding bulk solvent, including some human biliverdin reductases and nitric oxide synthase. ${ }^{42-44}$

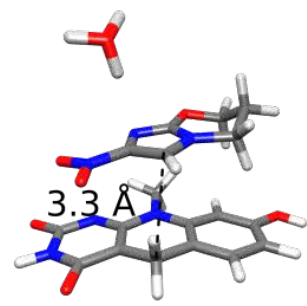

$\mathrm{RC}$

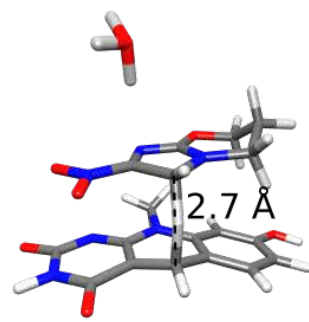

TS1

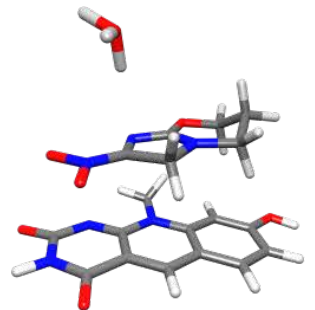

IC1

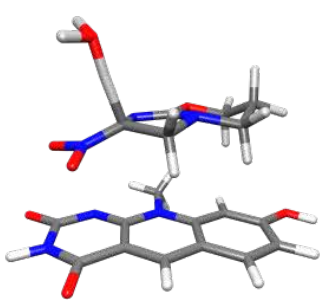

TS2

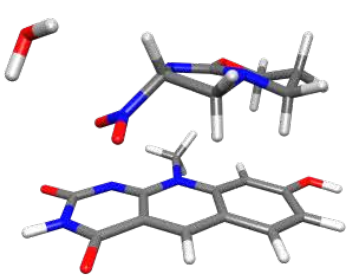

IC2

Figure 4. The optimized geometries of the truncated $\mathrm{F}_{420} \mathrm{H}^{-}$and pretomanid species used for DFT calculations of the reaction profile. RC was initially obtained from the energy minimized crystal structure conformation, as used to initiate the MD simulations. A water molecule was replaced with a hydroxonium ion and optimized. 


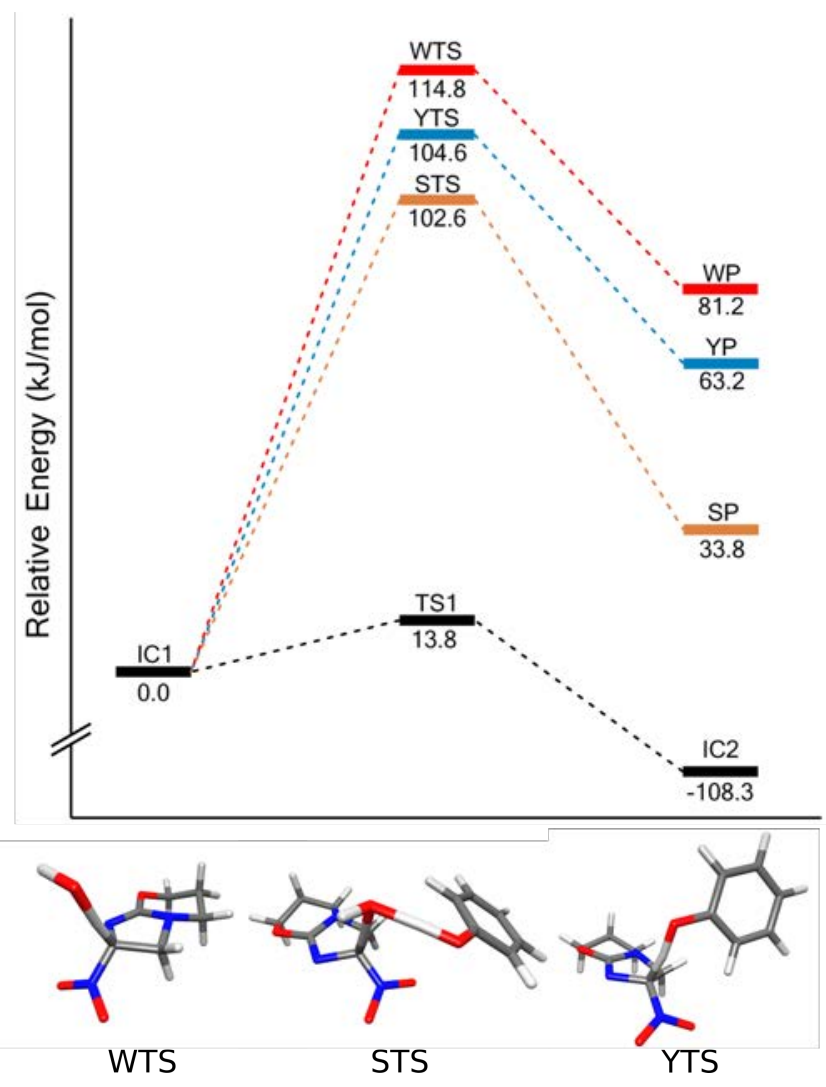

Figure 5. Comparison of the energy profiles for the alternate proton transfer reactions: $\mathrm{H}_{3} \mathrm{O}^{+}$(TS1), water (WTS), tyrosine (YTS) and tyrosine assisted (STS). The optimized transition state structures for the three latter mechanisms are also shown while the transition state for $\mathrm{H}_{3} \mathrm{O}^{+}$was shown previously in Figure 4 .

Mechanism (B). In this mechanism, the proton donor is a neutral water molecule. The transition state for this proton transfer, WTS, has a high barrier of $114.8 \mathrm{~kJ} \mathrm{~mol}^{-1}$. Such high energy barriers for proton transfer from neutral water have previously been reported for other enzyme mechanisms where hydride transfer and proton transfer occur. ${ }^{43,44}$ The reaction is not energetically favourable, with the resulting product WP $81.2 \mathrm{~kJ} \mathrm{~mol}^{-1}$ higher in energy than IC1.

Mechanism (C). The third mechanism used the hydroxyl group on a nearby tyrosine, most likely Tyr130 based on existing mutagenesis data, ${ }^{15}$ to assist in the proton transfer from a neutral water molecule in a concerted step. This mechanism is plausible, since the Tyr130 hydroxyl group is in the correct vicinity to align a water molecule to the $\mathrm{C} 2$ atom of pretomanid. Furthermore, in previous studies, mutation of Tyr130 to Phe130, which loses the hydroxyl group but keeps the aromatic ring intact, leads to a loss of approximately half of the enzyme's catalytic activity. ${ }^{15}$ The energy barrier to the transition state STS in this reaction was calculated to be $102.6 \mathrm{~kJ}$ . $\mathrm{mol}^{-1}$. This is lower than the reaction with neutral water at $114.8 \mathrm{~kJ} \mathrm{~mol}^{-1}$, but still much higher than for mechanism (A). The product SP formed from this reaction is $33.8 \mathrm{~kJ} \mathrm{~mol}^{-1}$ higher than the starting complex IC1. This is lower than in mechanism (B), but still energetically unfavorable.

Mechanism (D). The final mechanism considered the possibility of the hydroxyl group of a tyrosine residue being the direct proton donor in the reaction with no involvement from water. Tyrosine can act as a weak biological acid and has been known to act as a proton donor in other enzyme mechanisms. ${ }^{45}$
For this reaction, the relative energy of the transition state YTS was calculated to be $104.6 \mathrm{~kJ} \mathrm{~mol}^{-1}$. This barrier is again lower than the barrier in mechanism (B) and close to the barrier for the concerted transfer in mechanism (C), although it is much less energetically favourable than $(\mathbf{C})$, with a reaction energy of $63.2 \mathrm{~kJ} \mathrm{~mol}^{-1}$.

On the basis of Figure 5, it would thus seem that the hydroxonium ion is the most likely proton donor. In order to further test this, $100 \mathrm{~ns}$ MD simulations were used to study the accessibility and movement of water molecules within the active site. The radial distribution function (RDF) of water molecules around $\mathrm{C} 2$ atom of pretomanid was calculated from MD trajectories to examine the hydration of the active site. As implemented here, the RDF calculates the probability of finding a water molecule within a specified distance from the reference point, $\mathrm{C} 2$. The RDF of water in the simulations used to study the initial reaction complex was calculated to have a small peak at $4.75 \AA$, corresponding to the distance from $\mathrm{C} 2$ to the closest water oxygen. This is too large a distance for effective proton transfer to take place and arises from the aforementioned hydrophobic barrier formed by the Tyr65, Tyr130, Tyr136 residues that excluded the solvent from the active site.

MD simulations were also performed with the charged intermediate species of pretomanid present after hydride transfer (as in IC1 in Figure 4). Since the proton transfer step likely takes place following hydride transfer, the new intermediate might result in significant changes to the behavior of the solvent and the active site residues. The RDF of water with $\mathrm{C} 2$ of the charged intermediate of pretomanid as a reference is compared with the RDF in the initial reaction complex in Figure 6A. The RDF with the charged species exhibited a higher probability peak at a closer distance of $3.75 \AA$. This improved water accessibility is facilitated by the disruption of the hydrophobic tyrosine barrier responsible for water exclusion. Cluster analysis was used to sort the frames of the simulation into clusters of similar structures based on their RMSD values to find the most populated conformation of the active site. In the representative conformation of the largest cluster was found that Tyr136 shifted away from its original conformation overhanging the nitronate head group of pretomanid, as shown in Figure 6C. This movement led to the disruption of the hydrophobic barrier and increased water accessibility to facilitate proton transfer. This altered conformation was present for $25 \%$ of the simulation with the charged intermediate whereas it was absent in the MD simulations with the initial reaction complex. The increased water accessibility from this conformational change increases the likelihood that hydroxonium or a (protonated) water cluster could act as the proton donor and follow the low reaction barrier pathway of mechanism (A). ${ }^{46}$

\subsection{Step 3: Intra-Molecular Decomposition}

The final step of the activation reaction involves decomposition of the pretomanid intermediate in IC2 formed through previous two steps, yielding the final products, desnitroimidazole and nitrous acid. This process involves intramolecular rearrangement in the pretomanid intermediate in IC2, independent of the presence of the $F_{420}$ and the proton donor. Hence, Model 3, consisting only of the reduced pretomanid intermediate in IC2 and an ethanol molecule as a serine (Ser78) analogue, was used in the calculations to characterize the reaction profile for this step. The relative energies were calculated with respect to the new starting point (Model 3). In our proposed mechanism, the intramolecular decomposition 
begins with the cleavage of the $\mathrm{C}-\mathrm{N}$ bond between the newly protonated $\mathrm{C} 2$ atom and the nitronate group. The energy barrier for this step is $50.8 \mathrm{~kJ} \mathrm{~mol}^{-1}$ (TS3), followed by formation of an intermediate complex (IC3) between carbocation desnitro species and $\mathrm{NO}_{2}^{-}$. This intermediate is $30.7 \mathrm{~kJ} \mathrm{~mol}^{-1}$ higher in energy than IC2.

Finally, nitrous acid $\left(\mathrm{HNO}_{2}\right)$ is formed via proton abstraction by the $\mathrm{NO}_{2}^{-}$ion from the $\mathrm{C} 5$ atom, resulting in restoration of the double bond between $\mathrm{C} 4$ and $\mathrm{C} 5$ of the des-nitro species. The transition state TS4 for this proton transfer is $67.1 \mathrm{~kJ} \mathrm{~mol}^{-}$ ${ }^{1}$ higher than starting complex IC2, and the final product com- plex $\mathbf{P C}$ is $7.8 \mathrm{~kJ} \mathrm{~mol}^{-1}$ higher than the starting point (IC2). Inclusion of an ethanol molecule to represent Ser78, which forms hydrogen bond with the nitronate group (Model 3A), changes the reaction profile. The reaction barrier for TS3 is slightly lower $\left(45.4 \mathrm{~kJ} \mathrm{~mol}^{-1}\right)$, however, the intermediate IC3 is significantly stabilized by $18.7 \mathrm{~kJ} \mathrm{~mol}^{-1}$ in the presence of this hydrogen bond. Expectedly, this hydrogen bond increased the energy for the proton abstraction by $\mathrm{NO}_{2}^{-}$ion from $\mathrm{C} 5$ atom by $12.8 \mathrm{~kJ} \mathrm{~mol}^{-1}$, while lowering the energy of the final product complex (PC) by $14.4 \mathrm{~kJ} \mathrm{~mol}^{-1}$ compared to the profile obtained without the serine mimic (Model 3).

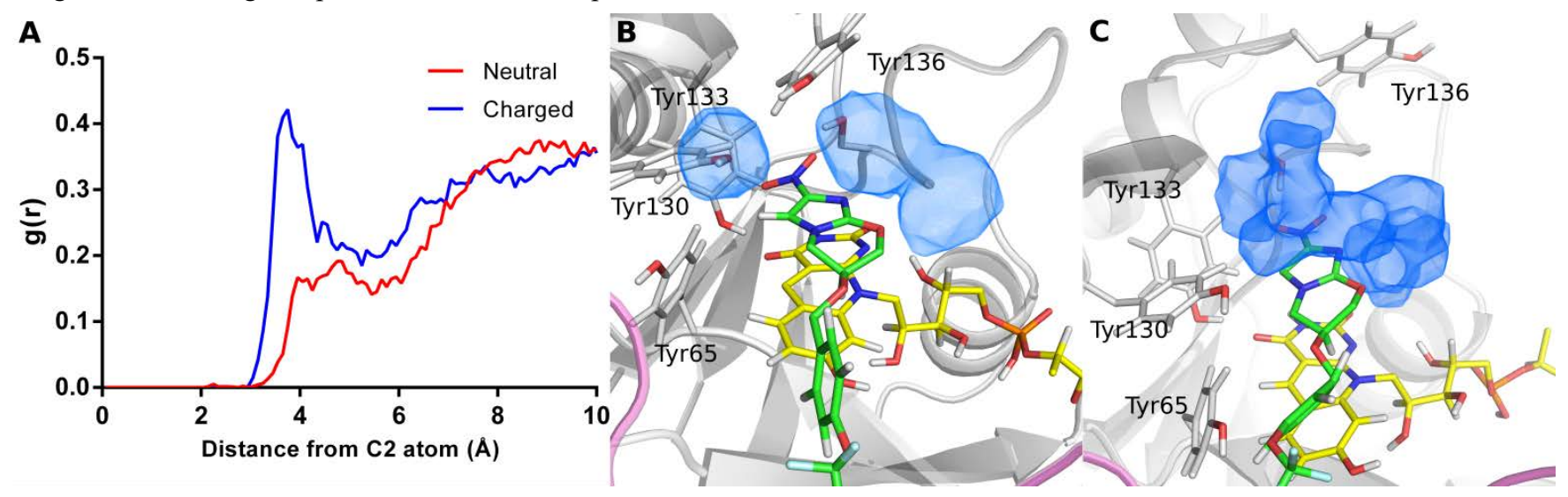

Figure 6. A. Plot of the radial distribution function (RDF) for water around the $\mathrm{C} 2$ atom of pretomanid. The charged intermediate (blue) exhibits a much higher probability of water being found in close proximity of C2 with a peak at $3.75 \AA$ compared to the neutral substrate (red) which has an lower probability peaking at $4.75 \AA$. B. The initial closed conformation of Tyr136 with the density of water molecules within $6 \AA$ of $\mathrm{C} 2$ displayed as an isomesh in blue. C. The open conformation of Tyr136 increases the density of water molecules in the area immediately above $\mathrm{C} 2$ of pretomanid

Scheme 3. The complete reaction mechanism for the activation of pretomanid by Ddn using $\mathbf{F}_{420} \mathbf{H}_{2}$.<smiles></smiles>

RC<smiles>CN1C2=NC(=O)C(=O)NC(=O)C2=Cc2c([N+](=O)[O-])nc3n2CC1CO3</smiles>

TS1<smiles></smiles>

IC1<smiles>[2H]C1N2CC(C)COC2=N[C@]1([PH3+])[N+](=O)[O-]</smiles>

TS2<smiles>Cc1cn2c(n1)OCCC2</smiles>

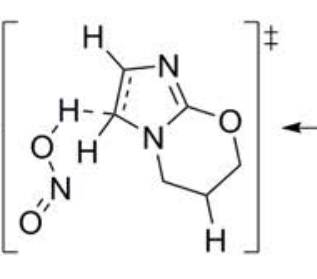<smiles>CCC[C@H]1CCCCO1</smiles><smiles>[3H]C1COC2=N[C@H]([N+](=O)[O-])[C@H](C)N2C1</smiles><smiles>[C+]C(C)C</smiles><smiles>O=[N+]([O-])[C@@H]1CN2CCCOC2=N1</smiles> 


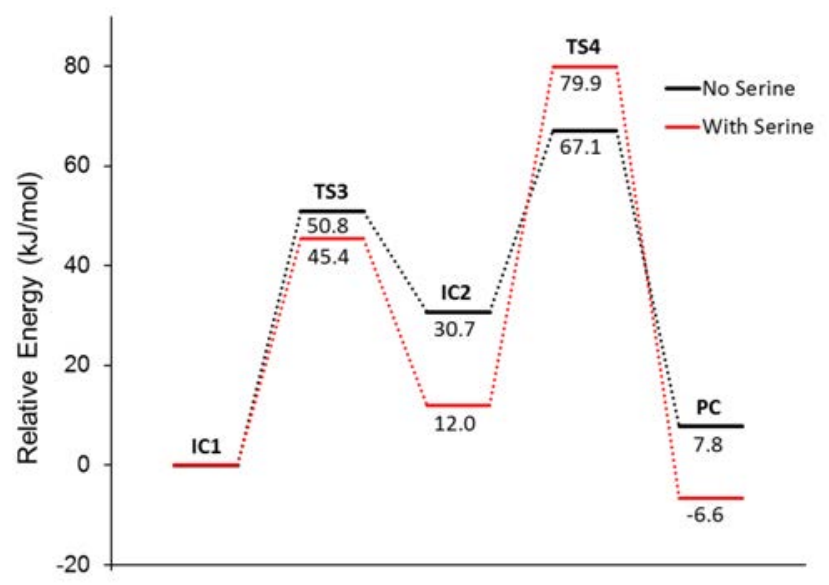

Figure 7. The energy profile diagram for the pretomanid decomposition mechanism using Model 3 and $3 \mathrm{~A}$ in an aqueous environment. The final product $\mathbf{P C}$ is the lowest point on the energy profile diagram suggesting that the reaction is energetically favourable.

\section{DISCUSSION}

While residues Ser78 and Tyr133 were observed to function in their predicted roles by hydrogen bonding with pretomanid and $\mathrm{F}_{420} \mathrm{H}^{-}$respectively to align the Michaelis complex, ${ }^{15} \mathrm{MD}$ simulations suggest the roles of Tyr65, Tyr130 and Tyr136 are also worth noting. When studied individually, the three tyrosine residues were not observed in the MD simulations to be involved in substrate or cofactor binding, aside from some possible $\pi$-stacking interactions between Tyr136 and pretomanid. However, together, the three tyrosine residues form a hydrophobic barrier spanning from the hydride transfer site at $\mathrm{C} 5$ of $\mathrm{F}_{420} \mathrm{H}^{-}$and $\mathrm{C} 3$ of pretomanid, to the nitronate group and $\mathrm{C} 2$ of pretomanid. This hydrophobic barrier is strengthened by hydrogen bonding interactions between the hydroxyl groups of Tyr65, Tyr130 and Tyr136, although these interactions are intermittent. This hypothesis is complemented by published mutagenesis results, which show that upon mutation of any of these residues to phenylalanine, which contains the aromatic ring but not the hydroxyl group, ${ }^{15}$ catalysis still takes place although at a lower rate. Conversely, mutation of these residues to non-aromatic amino acids such as leucine, valine or alanine resulted in complete loss of activity, indicating that the aromatic groups are crucial for catalysis and the hydroxyl groups in tyrosine may provide a means to strengthen the barrier alignment.

Comparison of the reaction profile for hydride transfer (Figure 3 ) calculated in water and toluene showed that the change in the solvent polarity affects the relative energy of the formed intermediate (IC1) stronger than the reaction barrier. The intermediate IC1 is $77 \mathrm{~kJ} \mathrm{~mol}^{-1}$ lower in energy than in toluene, but MD simulations with IC1 in the active site resulted with an increased solvent accessibility and this change in the local polarity could have stabilizing effect for this intermediate. Pretomanid is additionally hydrogen bonded to Ser78, which could also lead to catalytic acceleration. These types of interactions are further strengthened in (desolvated) low polarity environments, which explain the catalytic importance of the aromatic hydrophobic barrier. ${ }^{47}$ Similar hydrophobic shielding of the hydride transfer site by a non-binding tyrosine residue was also observed in our recent work characterizing Rv2074 which is an $\mathrm{F}_{420} \mathrm{H}_{2}$-dependent biliverdin reductase found in $M$. tuberculosis. ${ }^{48}$ This type of hydrophobic pockets seems to be a common feature in flavoenzymes catalyzing hydride transfer, ${ }^{49}$ but whether the role of these residues goes beyond protecting the active site from solvent is not entirely clear.

The reaction mechanism proceeds via an initial hydride transfer step followed by proton transfer after which the pretomanid product undergoes intramolecular decomposition to produce the final products. Using QM calculations, the ratedetermining step of this mechanism was calculated to be the hydride transfer step, with a calculated relative energy barrier of $95.8 \mathrm{~kJ} \mathrm{~mol}^{-1}$ in aqueous and $117.8 \mathrm{~kJ} \mathrm{~mol}^{-1}$ in the toluene solvent model. As determining the exact dielectric constant within an enzyme active site is extremely difficult, these two scenarios represent the two extremes with dielectric constants of 80.1 and 2.38 respectively. Although this barrier range is much higher than any of the following steps in the reaction, it is consistent with barriers for other enzyme mechanisms involving hydride transfer. ${ }^{50}$ The energy barrier could additionally be inflated due to other contributing factors stemming from the methods used. For example, the level of theory used in this study, M06-2X, has been reported with errors of $4-7$ $\mathrm{kJ} \mathrm{mol}^{-1}$ for barrier calculations involving hydrogen transfer. ${ }^{51,52}$

An important contributing factor to the high barrier could be the exclusion of stabilization provided by the surrounding enzyme residues in the active site in our QM structures. Studies of other enzyme mechanisms involving comparisons of reaction barriers between quantum cluster and QM/MM methods have shown activation barriers that were $12 \mathrm{~kJ} \mathrm{~mol}^{-1}$ to 40 $\mathrm{kJ} \mathrm{mol}^{-1}$ lower in the QM/MM treatment. ${ }^{53,54}$ The lower barriers in these studies are attributed to long range electrostatic interactions between the QM and MM regions influencing the energies of the charged intermediates and transition states, as well as conformational reorganization due to charge redistribution. ${ }^{53}$ Our system involves charge transfer and redistribution. It is likely that the interactions between the protein and the reactants will affect the reaction profile, enabling successful catalysis. This is clearly demonstrated by the effect of the inclusion of Ser78 mimic in the calculations for Step 3, showing how the presence of hydrogen bonding can shift the relative energies of the transition states and intermediate structures. Similarly, a test calculation performed with ethanol as a model for Ser78 lowered the barrier for the hydride transfer step by $7 \mathrm{~kJ} \mathrm{~mol}^{-1}$, Ser78 was implicated as a crucial residue in the mutation studies, rendering the enzyme inactive if substituted by alanine.

The use of a hydroxonium ion as a proton donor in proton transfer has been characterized in many enzyme mechanisms. ${ }^{42-44}$ The alternate pathways involving tyrosine and neutral water all exhibited relatively high barriers at greater than $100 \mathrm{~kJ} \mathrm{~mol}^{-1}$ compared to the $4.6 \mathrm{~kJ} \mathrm{~mol}^{-1}$ barrier with the hydroxonium ion. The direct involvement of Tyr130 is also unlikely, since the Tyr130Phe mutation did not lead to complete loss of activity although it did reduce the rate by approximately $50 \%{ }^{15}$ This loss in activity can be attributed to changes in the previously discussed hydrophobic barrier, instead of a role in proton transfer. Generation of this hydroxonium species likely occurs by proton shuttling between the water molecules via the Grotthuss mechanism. It is possible that a protonated water cluster serves as a proton source, as found in bacteriorhodopsin. ${ }^{55,56}$ The excess protons could orig- 
inate from the $\mathrm{N} 1$ deprotonation of $\mathrm{F}_{420} \mathrm{H}_{2}$ at physiological $\mathrm{pH}$ values of 7.5. MD results indicate that the solvent accessibility of the active site increases upon hydride transfer, where movement of Tyr136 (Figure 6B and 6C) opens the substrate to a column of water molecules, which extends into the bulk solvent outside the enzyme. Tyrosine residues could have an additional role in positioning the proton source before transfer takes place.

\section{CONCLUSIONS}

In this study, we used a combination of QM and MD to investigate the reaction mechanism for the activation of pretomanid by Ddn and $\mathrm{F}_{420} \mathrm{H}^{-}$. Small quantum cluster models were used to characterize three distinct steps in the catalyzed reaction. The results are consistent with experimental data that suggests the rate-determining step for the reaction is the initial hydride transfer, while the subsequent protonation is most likely to occur from the solvent via proton shuttling. Furthermore, the modelling of the active site residues allowed assignment of plausible roles for residues surrounding the active site. MD simulations suggest that three tyrosine residues were found to form a hydrophobic barrier around the active site during the hydride transfer, and that this hydrophobic barrier can be disrupted upon substrate reduction to allow increased water accessibility to facilitate the subsequent proton transfer step. This analysis advances our understanding of the catalytic mechanism used by Ddn and will further our understanding of the wider FDOR super-family. Given the pivotal role that Ddn plays in activation of antimycobacterial prodrugs, the new insight presented here could aid in the development of new treatments involving mycobacterial pathogens.

\section{ASSOCIATED CONTENT}

Supporting Information. Further data, including an assessment of theoretical procedures, an MD analysis of an alternative binding mode in the active site, a QM analysis of the role of Ser 78, and geometries of all species. This material is available free of charge via the Internet at http://pubs.acs.org.

\section{AUTHOR INFORMATION}

\section{Corresponding Author}

* Email: michelle.coote $@$ anu.edu.au (M.L.C.) colin.jackson@anu.edu.au (C.J.J.)

\section{Author Contributions}

The manuscript was written through contributions of all authors.

\section{Funding Sources}

Australian Research Council (grant number DP130102144)

\section{ACKNOWLEDGMENT}

MLC and CJJ gratefully acknowledge support from the Australian Research Council under their Discovery Projects program. MLC also gratefully acknowledges generous allocations of supercomputing time on the National Facility of the Australian National Computational Infrastructure.

\section{REFERENCES}

2012, $36(3), 514$

Flynn, J. L.; Chan, J. Infect. Immun. 2001, 69 (7), 4195.

Zhang, Q.; Xiao, H.-P.; Cui, H.-Y.; Sugawara, I. J. Int. Med. Res. 2011, 39 (1), 105.

Jain, S. K.; Lamichhane, G.; Nimmagadda, S.; Pomper, M. G.; Bishai, W. R. 2008, 285.

Stover, C. K.; Warrener, P.; VanDevanter, D. R.; Sherman, D. R.; Arain, T. M.; Langhorne, M. H.; Anderson, S. W.; Towell, J. A.; Yuan, Y.; McMurray, D. N.; Kreiswirth, B. N.; Barry, C. E.; Baker, W. R. Nature 2000, 405 (6789), 962.

Blair, H. A.; Scott, L. J. Drugs 2015, 75 (1), 91.

Tasneen, R.; Williams, K.; Amoabeng, O.; Minkowski, A.; Mdluli, K. E.; Upton, A. M.; Nuermberger, E. L. Antimicrob. Agents Chemother. 2015, 59 (1), 129.

Singh, R.; Manjunatha, U.; Boshoff, H. I.; Ha, Y. H.; Niyomrattanakit, P.; Ledwidge, R.; Dowd, C. S.; Lee, I. Y.; Kim, P.; Zhang, L.; Kang, S.; Keller, T. H.; Jiricek, J.; Barry 3rd, C. E. Science (80-. ). 2008, 322 (5906), 1392.

Manjunatha, U.; Boshoff, H. I. M.; Barry, C. E. Commun. Integr. Biol. 2009, 2 (3), 215.

Ahmed, F. H.; Carr, P. D.; Lee, B. M.; Afriat-Jurnou, L.; Mohamed, A. E.; Hong, N.-S.; Flanagan, J.; Taylor, M. C.; Greening, C.; Jackson, C. J. J. Mol. Biol. 2015, 427 (22), 3554

Mohamed, A. E.; Ahmed, F. H.; Arulmozhiraja, S.; Lin, C. Y.; Taylor, M. C.; Krausz, E. R.; Jackson, C. J.; Coote, M. L. Mol. Biosyst. 2016, 12 (4), 1110.

Jacobson, F.; Walsh, C. Biochemistry 1984, 23 (5), 979.

Purwantini, E.; Daniels, L. J. Bacteriol. 1996, 178 (10), 2861.

Cellitti, S. E.; Shaffer, J.; Jones, D. H.; Mukherjee, T.; Gurumurthy, M.; Bursulaya, B.; Boshoff, H. I.; Choi, I.; Nayyar, A.; Lee, Y. S.; Cherian, J.; Niyomrattanakit, P.; Dick, T.; Manjunatha, U. H.; Barry III, C. E.; Spraggon, G.; Geierstanger, B. H. Structure 2012, 20 (1), 101.

Biasini, M.; Bienert, S.; Waterhouse, A.; Arnold, K.; Studer, G.; Schmidt, T.; Kiefer, F.; Gallo Cassarino, T.; Bertoni, M.; Bordoli, L.; Schwede, T. Nucleic Acids Res. 2014, 42 (Web Server issue), W252.

Arnold, K.; Bordoli, L.; Kopp, J.; Schwede, T. Bioinformatics 2006, 22 (2), 195.

Kiefer, F.; Arnold, K.; Künzli, M.; Bordoli, L.; Schwede, T. Nucleic Acids Res. 2009, 37 (Database issue), D387.

Guex, N.; Peitsch, M. C.; Schwede, T. Electrophoresis 2009, 30 (S1), S162.

Hess, B.; Kutzner, C.; van der Spoel, D.; Lindahl, E. J. Chem. Theory Comput. 2008, 4 (3), 435.

Trott, O.; Olson, A. J. J. Comput. Chem. 2010, 31 (2), 455.

Morris, G. M.; Huey, R.; Lindstrom, W.; Sanner, M. F.; Belew, R. K.; Goodsell, D. S.; Olson, A. J. J. Comput. Chem. 2009, 30 (16), 2785.

Koziara, K. B.; Stroet, M.; Malde, A. K.; Mark, A. E. J. Comput. Aided. Mol. Des. 2014, 28 (3), 221.

Schmid, N.; Eichenberger, A. P.; Choutko, A.; Riniker, S.; Winger, M.; Mark, A. E.; van Gunsteren, W. F. Eur. Biophys. J. 2011, 40 (7), 843 . 
(26) Hess, B.; Bekker, H.; Berendsen, H. J. C.; Fraaije, J. G. E. M. J Comput. Chem. 1997, 18 (12), 1463.

(27) Berendsen, H. J. C.; Postma, J. P. M.; van Gunsteren, W. F.; DiNola, A.; Haak, J. R. J. Chem. Phys. 1984, 81 (8), 3684.

(28) Smith, P. E.; van Gunsteren, W. F. Chem. Phys. Lett. 1993, 215 (4), 315 .

(29) Daura, X.; Gademann, K.; Jaun, B.; Seebach, D.; van Gunsteren, W. F.; Mark, A. E. Angew. Chemie Int. Ed. 1999, 38 (1-2), 236.

(30) Humphrey, W.; Dalke, A.; Schulten, K. J. Mol. Graph. 1996, 14 (1), 33 .

(31) Sousa, S. F.; Fernandes, P. A.; Ramos, M. J. Phys. Chem. Chem. Phys. 2012, 14 (36), 12431.

(32) Curtiss, L. A.; Redfern, P. C.; Raghavachari, K.; Rassolov, V.; Pople, J. A. J. Chem. Phys. 1999, 110 (10), 4703.

Curtiss, L. A.; Raghavachari, K.; Redfern, P. C.; Baboul, A. G.; Pople, J. A. Chem. Phys. Lett. 1999, 314 (1), 101.

Frisch, M. J.; Trucks, G. W.; Schlegel, H. B.; Scuseria, G. E.; Robb, M. A.; Cheeseman, J. R.; Scalmani, G.; Barone, V.; Mennucci, B.; Petersson, G. A.; Nakatsuji, H.; Caricato, M.; Li, X.; Hratchian, H. P.; Izmaylov, A. F.; Bloino, J.; Zheng, G.; Sonnenberg, J. L.; Hada, M.; Ehara, M.; Toyota, K.; Fukuda, R.; Hasegawa, J.; Ishida, M.; Nakajima, T.; Honda, Y.; Kitao, O.; Nakai, H.; Vreven, T.; Montgomery Jr., J. A.; Peralta, J. E.; Ogliaro, F.; Bearpark, M. J.; Heyd, J.; Brothers, E. N.; Kudin, K. N.; Staroverov, V. N.; Kobayashi, R.; Normand, J.; Raghavachari, K.; Rendell, A. P.; Burant, J. C.; Iyengar, S. S.; Tomasi, J.; Cossi, M.; Rega, N.; Millam, N. J.; Klene, M.; Knox, J. E.; Cross, J. B.; Bakken, V.; Adamo, C.; Jaramillo, J.; Gomperts, R.; Stratmann, R. E.; Yazyev, O.; Austin, A. J.; Cammi, R.; Pomelli, C.; Ochterski, J. W.; Martin, R. L.; Morokuma, K.; Zakrzewski, V. G.; Voth, G. A.; Salvador, P.; Dannenberg, J. J.; Dapprich, S.; Daniels, A. D.; Farkas, Ö.; Foresman, J. B.; Ortiz, J. V; Cioslowski, J.; Fox, D. J. Gaussian, Inc.: Wallingford, CT, USA 2009,

(35) Marenich, A. V; Cramer, C. J.; Truhlar, D. G. J. Phys. Chem. B 2009, 113 (18), 6378.

(36) Hratchian, H. P.; Schlegel, H. B. J. Chem. Phys. 2004, 120 (21) 9918.

(37) Hratchian, H. P.; Schlegel, H. B. J. Chem. Theory Comput. 2005, 1 (1), 61 .

(38) Shima, S.; Warkentin, E.; Grabarse, W.; Sordel, M.; Wicke, M.; Thauer, R. K.; Ermler, U. J. Mol. Biol. 2000, 300 (4), 935.
Shima, S.; Ermler, U. Biochemistry 2009, 48 (42), 10098.

Hammes-Schiffer, S.; Watney, J. B. Philos. Trans. R. Soc. Lond. B. Biol. Sci. 2006, 361 (1472), 1365.

Gurumurthy, M.; Mukherjee, T.; Dowd, C. S.; Singh, R.; Niyomrattanakit, P.; Tay, J. A.; Nayyar, A.; Lee, Y. S.; Cherian, J.; Boshoff, H. I.; Dick, T.; Barry, C. E.; Manjunatha, U. H. FEBS J. 2012, 279 (1), 113.

Fu, G.; Liu, H.; Doerksen, R. J. J. Phys. Chem. B 2012, 116 (32), 9580.

Smith, L. J.; Browne, S.; Mulholland, A. J.; Mantle, T. J. Biochem. J. 2008, 411 (3), 475.

Cho, K.-B.; Derat, E.; Shaik, S. J. Am. Chem. Soc. 2007, 129 (11), 3182 .

Bohren, K. M.; Grimshaw, C. E.; Lai, C. J.; Harrison, D. H.; Ringe, D.; Petsko, G. A.; Gabbay, K. H. Biochemistry 1994, 33 (8), 2021.

Garczarek, F.; Brown, L. S.; Lanyi, J. K.; Gerwert, K. Proc. Natl. Acad. Sci. U. S. A. 2005, 102 (10), 3633.

Richard, J. P.; Amyes, T. L.; Goryanova, B.; Zhai, X. Curr. Opin. Chem. Biol. 2014, 21, 1.

Ahmed, F. H.; Mohamed, A. E.; Carr, P. D.; Lee, B. M.; Condic-Jurkic, K.; O’Mara, M. L.; Jackson, C. J. Protein Sci. 2016, 25(9), 1692.

Fraaije, M. W.; Mattevi, A. Trends Biochem. Sci. 2000, 25 (3), 126.

Ion, B. F.; Bushnell, E. A. C.; Luna, P. De; Gauld, J. W. Int. J. Mol. Sci. 2012, 13 (12), 12994.

Zhao, Y.; Truhlar, D. G. Theor. Chem. Acc. 2008, 119 (5-6), 525 .

Cohen, A. J.; Mori-Sánchez, P.; Yang, W. Chem. Rev. 2012, 112 (1), 289 .

Liao, R.-Z.; Thiel, W. J. Chem. Theory Comput. 2012, 8 (10), 3793 .

Siegbahn, P. E. M.; Borowski, T. Faraday Discuss. 2011, 148 (0), 109.

Rousseau, R.; Kleinschmidt, V.; Schmitt, U. W.; Marx, D. Phys. Chem. Chem. Phys. 2004, 6 (8), 1848.

Morgan, J. E.; Vakkasoglu, A. S.; Lanyi, J. K.; Lugtenburg, J.; Gennis, R. B.; Maeda, A. Biophys. J. 2012, 103 (3), 444. 
Authors are required to submit a graphic entry for the Table of Contents (TOC) that, in conjunction with the manuscript title, should give the reader a representative idea of one of the following: A key structure, reaction, equation, concept, or theorem, etc., that is discussed in the manuscript. Consult the journal's Instructions for Authors for TOC graphic specifications.

Insert Table of Contents artwork here

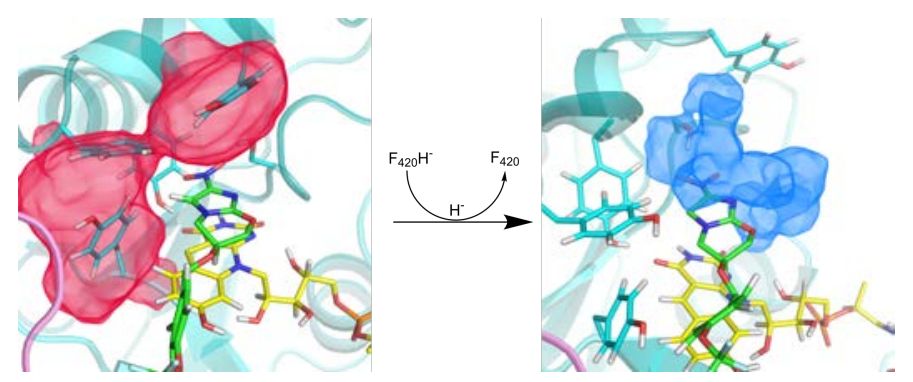

